Historic, Archive Document

Do not assume content reflects current scientific knowledge, policies, or practices. 


$$
\text { . }
$$




\section{Irice List}

COTTON PLANTHA SEED

Fall 1929 and Spring 1930

\section{Delfos-6102-A2-C3}

SACK LOTS (100 its $)$ $\$ 6.00$

TON LOTS 110.00

CAR LOTS (20 Tons) PER TON 100.00

Delfos $-6102-911$

SACK LOTS (100 its)

TON LOTS

CAR LOTS (20 Tons) PER TON

Delfos-6102-0245

SACK LOTS (100 $\mathrm{Hs})$ 110.00

TON LOTS

CAR LOTS (20 Tons) PER TON S 7.00 125.00 115.00

\section{Delfos No. 2}

SACK LOTS (100 $\mathrm{tbs}) \ldots$ \& 7.00 TON LOTS

CAR LOTS (20 Tons) PER TON 125.00

Delfos-6102-531

$$
\text { And }
$$

Delfos-6102-550

SACK LOTS (100 tos) $\$ \quad 7.50$ 7.50
135.00 TON LOTS

CAR LOTS (20 Tons) PER TON 125.00

\section{Delfos-631- 463}

SACK LOTS (100 ibs) $\$ 9.00$

TON LOTS

CAR LOTS (20 Tons) PER TON

\section{Stoneville No. 1

$$
\text { And }
$$ \\ Stoneville No. 2}

SACK LOTS (100 Ibs)

TON LOTS

CAR LOTS (20 TONS) PER TON

165.00

All Prices F. O. B. Cars, Leland, Miss.

Above Prices Subject to Change IVithout Notice CASH WITH ORDER OR SIGHT DRAFT BILL OF LADING ATTACHED

\section{Stoneville Pedigreed Seed Co.}

Stoneville, Mississippi 


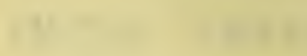

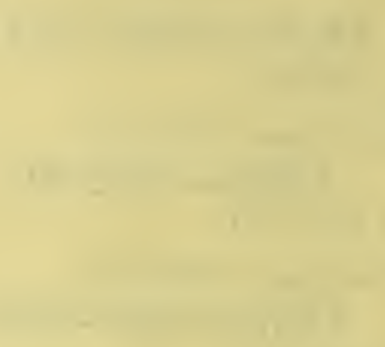

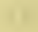

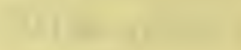

$-5$ 